\title{
Post-operative brachial plexus neuropraxia: A less recognised complication of combined plastic and laparoscopic surgeries
}

\author{
Jimmy Thomas \\ Department of Plastic Surgery, Division of Burns, Al Wakra Hospital, Al Wakra, Qatar
}

Address for correspondence: Dr. Jimmy Thomas, Department of Plastic Surgery, Division of Burns, Al Wakra Hospital, P O Box: 82228 , Al Wakra, Qatar. E-mail: jtjimmy@hotmail.com

\begin{abstract}
This presentation is to increase awareness of the potential for brachial plexus injury during prolonged combined plastic surgery procedures. A case of brachial plexus neuropraxia in a 26 -year-old obese patient following a prolonged combined plastic surgery procedure was encountered. Nerve palsy due to faulty positioning on the operating table is commonly seen over the elbow and popliteal fossa. However, injury to the brachial plexus has been a recently reported phenomenon due to the increasing number of laparoscopic and robotic procedures. Brachial plexus injury needs to be recognised as a potential complication of prolonged combined plastic surgery. Preventive measures are discussed.
\end{abstract}

\section{KEY WORDS}

Brachial plexus injury; laparoscopic procedure; liposuction

\section{INTRODUCTION}

case of brachial plexus injury after a combined plastic surgery procedure along with review of diagnosis and management is mentioned to highlight the relevance of positioning of the patient and monitoring during surgery.

\section{CASE REPORT}

A 26-year-old, obese lady, weighing 100 kilograms, and who had two caesarean sections and an abdominoplasty

\begin{tabular}{|l|l|}
\hline \multicolumn{2}{|c|}{ Access this article online } \\
\hline Quick Response Code: & Website: \\
\hline & www.ijps.org \\
\cline { 2 - 2 } & Dol: \\
\hline
\end{tabular}

in the past, was admitted for laparoscopic assisted vaginal hysterectomy along with liposuction of the anterior abdominal wall and flanks and repositioning of the neo umbilicus She was on medication for hypothyroidism and other than being obese, had no other comorbid factors.

During the initial laparoscopic procedure, the patient was placed in a steep Trendelenburg procedure with arms abducted to about $45^{\circ}$. To prevent the patient sliding off, a metallic device with two padded shoulder plates on either side of the neck, was attached to the operating table at the head end. The laparoscopic procedure lasted for $2^{1 / 2} \mathrm{~h}$. After this, the table was straightened, and the plastic surgery procedures completed. This lasted for $1 \frac{1}{2} \mathrm{~h}$ and the total duration of the procedures was more than $4 \mathrm{~h}$.

On the first post-operative day, the patient complained of burning pain along the outer aspect of the right arm and initially, this was thought to be due to the prolonged 
use of a non-invasive blood pressure cuff. However, by next morning, the patient had difficulty abducting the right shoulder. There was numbness along the thumb and index finger and the radial border of the forearm. Biceps flexion was absent with weakness of the triceps extension. The patient was assessed by a senior neurosurgeon, who confirmed the clinical diagnosis of upper brachial plexopathy. Magnetic resonance imaging (MRI) scan of the brain and spine showed only osteophytes of the C5, C6 level. Nerve conduction report was cryptic and showed absent conduction in the musculocutaneous nerve.

The patient was started on a course of physiotherapy with active and passive range of motion. She was also given oral Gabapentin and steroids (dexamethasone) along with non-steroidal analgesics. A protective broad arm sling was used to protect the flail limb. After 6 weeks of starting this regime, the nerve palsy recovered and by the $10^{\text {th }}$ week, she was back to using the limb normally.

\section{DISCUSSION}

Nerve palsy due to faulty positioning on the operating table resulting in prolonged pressure or compression or stretch of the nerves has been reported. ${ }^{[1,2]}$ It is commonly seen over the elbow and popliteal fossa. However, injury to the brachial plexus has been a recently reported phenomenon due to the increasing numbers of laparoscopic procedures being done nowadays. More reports have appeared in the laparoscopic and gynaecology literature than in the plastic surgery literature..$^{[3-7]}$ Hence, the reason for reporting this case and discussing possible causative factors and methods of prevention.

\section{Anatomy}

The brachial plexus supplies the upper limb and shoulder girdle, arising from the spinal roots C5-T1. These roots then merge to form three trunks-superior (C5, C6), middle (C7) and lower (C8, T1). Each trunk then splits into anterior and posterior divisions. These then regroup and form cords which give rise to the nerves of the upper limb-axillary, musculocutaneous, radial, median and ulnar nerves.

The brachial plexus is situated in the posterior triangle in the neck. It passes between the scalene muscles proximally and distally between the clavicle and first rib before passing into the arm. Thus, it can be seen that the plexus is slung between two fixed points, proximally at the vertebrae and the prevertebral fascia and distally it is attached to the axillary fascia.

Based on this anatomy and other external factors, Jackson and Keats ${ }^{[8]}$ from their cadaver studies postulated the following:

1. Extension of the head and neck with lateral flexion to the contralateral side widens the neck shoulder angle and stretches the plexus.

2. Local compression over the acromion or 2-3 inches medially by shoulder braces stretches the plexus under the depressed clavicle [Figure 1].

3. Lateral pressure of the shoulder with braces causes direct compression of the plexus by the humeral head [Figure 1].

4. Use of wrist bands to prevent patients from sliding off the table pulls the humeral head downward directly onto the plexus, thus compressing the plexus [Figure 1].

5. Abduction of more than $90^{\circ}$ of the externally rotated and extended arm stretches the plexus over the head of the humerus and the coracoid process [Figure 2].

Compression results in ischaemia by cutting off blood supply in the vasa nervosa. ${ }^{[5]}$ This is thought to be perhaps the most critical cause of brachial plexus injury. Nerve injury can be of three types. Neuropraxia is the mildest where the nerve and the axonal continuity are maintained, and there is only nerve demyelination. In axonotmesis, the continuity of the nerve sheath is maintained while there is a breakdown of the axonal continuity by Wallerian degeneration. In neurotmesis, both nerve sheath and the axonal continuity are disrupted. Brachial plexus injury can be of the upper roots $(\mathrm{C} 5, \mathrm{C} 6)$ or of the lower roots (C8. T1).

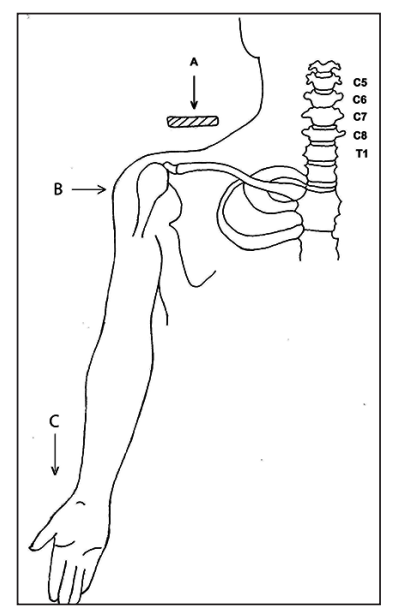

Figure 1: Line diagram showing pressure points (a) from shoulder blades (b) from humeral head (c) traction from wrist straps 
In the case presented, the patient was obese and her weight acting in conjunction with the resistance offered by the shoulder plates probably resulted in the upper brachial plexus injury [Figure 3]. Erb's point, formed by the union of $\mathrm{C} 5$, $\mathrm{C} 6$ nerves, is situated 2-3 inches above the mid-clavicular point. The long procedure time of $2 \frac{1}{2}$ $\mathrm{h}$ when this pressure was being exerted on the nerves may have also contributed to the prolonged ischaemic compression. This has been shown in the lower limbs placed in the lithotomy position. ${ }^{[3]}$

\section{Investigations}

Suspicion of brachial plexus injury in the post-operative period should arise when the patient complains of dysaesthesia, heaviness, weakness, and altered sensation in the affected limb. A thorough clinical history which includes details of the laparoscopic or robotic procedure should be taken. Clinical examination is detailing the sensory and motor loss should be meticulously done. An MRI scan may be helpful. Electrodiagnostic studies with nerve conduction and electromyography $(\mathrm{EMG})$ will help in diagnosing brachial plexus injury. ${ }^{[9]}$ Denervation changes in the nerves appear 2-3 weeks after the injury and hence an EMG study done immediately after the injury may not reveal the correct picture.

In the case presented, the nerve studies were done soon after the injury and hence probably did not reflect the full clinical picture. The report showed only delayed conduction in the musculocutaneous nerve. Lachman et al. have also reported a similar case of axonal injury in the musculocutaneous nerve. ${ }^{[10]}$ It is, therefore, recommended that the nerve studies be repeated after 4 weeks to elucidate any further changes.

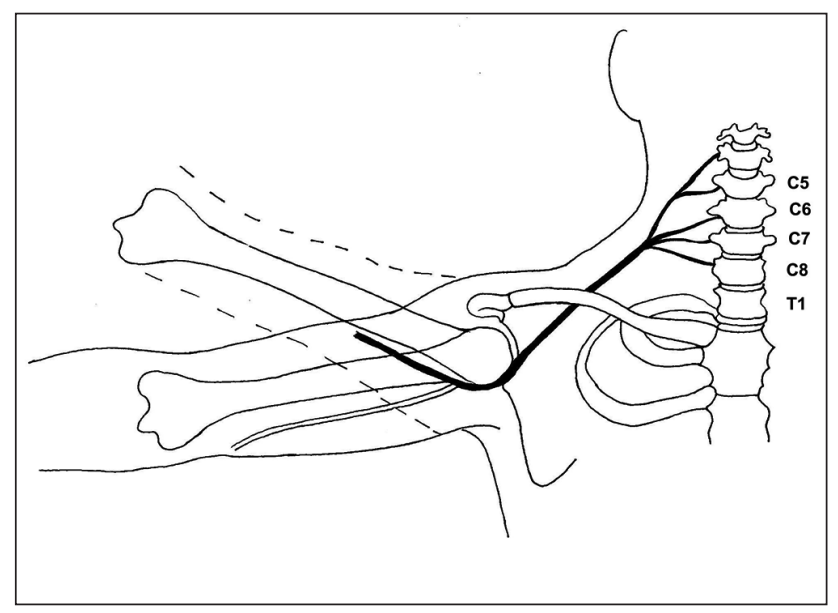

Figure 2: Line diagram showing brachial plexus (figurative) stretched over the head of the abducted humerus

\section{Treatment}

Treatment must be comprehensive and a multidisciplinary approach. There should be involvement of a neurologist and/or physical medicine specialist with other ancillary support.

Physiotherapy is the mainstay of treatment. The limb is put through the full range of passive motion to keep the joints supple. A protective splint is provided to prevent damage to the flail limb.

Pharmacologic agents used include Gabapentin, steroids and non-steroidal analgesics.

Gabapentin is an anti-epileptic agent, which has been found to be useful in chronic pain conditions, particularly neuropathic pain. In the oral form, Gabapentin is absorbed from the small intestine by facilitated transport and diffusion. Gabapentin is not metabolized in humans and is excreted without change in the urine. The exact mode of action of Gabapentin in neuropathic pain is not fully known. Gabapentin has been shown to inhibit ectopic discharge activity from damaged peripheral nerves. Gabapentin is well-tolerated, and the occasional sideeffects reported are sleepiness, dizziness, ataxia, and fatigue. The most serious of the reported side-effects was convulsions. Gabapentin is given orally thrice a day because of its short half-life. Recommended starting doses in neuropathic pain is $300 \mathrm{mg}$, and this can be increased to $3,600 \mathrm{mg} / \mathrm{day}$. The reason for using anticonvulsants in the treatment of neuropathic pain is based on similar events in the pathophysiology of epilepsy and neuropathic pain. ${ }^{[11]}$

According to the WHO, an adjuvant pain medication should be considered at all stages of the pain ladder. ${ }^{[12]}$

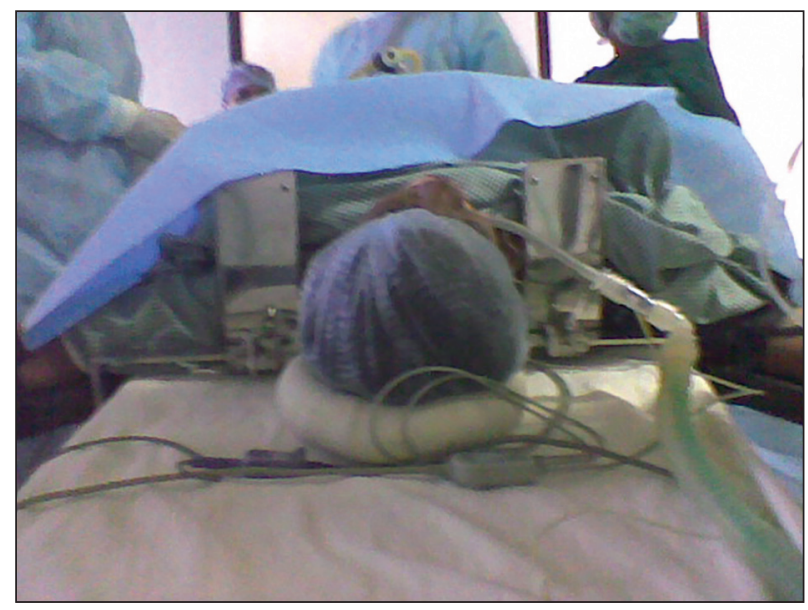

Figure 3: Shoulder blades in position over the plexus area 
Steroids are useful as adjuvant therapy for metastatic bone pain, neuropathic pain and visceral pain. ${ }^{[13]}$ Steroids, as adjuvants, help reduce opioid dose by acting in concert with opioids, reduce pain directly and have other beneficial symptomatic effects. The mechanism of action is by reducing spontaneous discharge in an injured nerve. They also reduce pain by inhibiting prostaglandin synthesis and reduce vascular permeability that causes tissue oedema. Dexamethasone is the most commonly used steroid for pain, but prednisolone also can be used. Dexamethasone has a longer half-life. A dose is $2-8 \mathrm{mg}$ thrice daily. After 2 weeks usage, it can be discontinued straightaway without any side-effects. Longer period of usage will require tapering of the dose prior to stopping it.

\section{Prognosis}

The prognosis of brachial plexus injury caused by positioning during surgery, is quite favourable. Most of the cases showed recovery by about 20 weeks. Diabetes mellitus was a cause of incomplete recovery. ${ }^{[14]}$

Operative time is a very major risk factor for brachial plexus injury, though no thresholds have been proven. The longer the duration of the surgery, the longer the ischaemia and thus the potential for nerve injury.

Very rarely nerve avulsion will require surgery.

\section{Prevention}

Prevention is based on principles emanating from understanding the anatomy and causative factors enunciated earlier.

1. The Trendelenburg position is adopted to allow the intestines to fall away from the pelvic cavity and thus allow visualisation of the pelvic organs. Maintaining the minimum gradient to allow for a smooth surgery will minimise chances of brachial plexus injury developing.

2. Keep the head and neck in a neutral position without allowing lateral flexion or dorsal extension.

3. Keeping the arms by the sides and avoiding arm boards which allow abduction, external rotation or hyperextension of the upper limb will reduce brachial plexus injury.

4. Avoid shoulder plates that cause local pressure over the brachial plexus and also wrist bands that pull the humeral head onto the brachial plexus.

5. Other methods used to prevent the patient sliding off the operating table include cross chest straps using foam material, ${ }^{[15]}$ gel mattresses which have high friction coefficient and eggshell foam. ${ }^{[16]}$

6. Perhaps the most important preventive measure is constant monitoring of the patient's positioning throughout the procedure.

7. More recently continuous monitoring of the somatosensory potentials in the ulnar nerve and transcranial electric motor evoked potentials have shown that impending brachial plexus injury can be detected earlier. This has been done more in relation to robotic thyroid surgery, but can be adopted in other cases as well. ${ }^{[17]}$

8. Karna et al. ${ }^{[18]}$ showed in their series that compression of the subclavian vessels can occur when the ribs are retracted upwards during hepatic surgeries. The compression occurs between the clavicle and first rib. This can result in compartment syndrome and to avoid this, they have suggested monitoring of the perfusion in both upper limbs using arterial line pressure measurements and pulse oximetry. This has been further endorsed by Thatte in his short and succinct commentary in the same issue. ${ }^{[19]}$

\section{SUMMARY}

Brachial plexus injury is happening after laparoscopic surgery is a rare complication. However, with increasing use of robotic surgery and combined procedures such as the one described in the case above, the incidence of this complication may increase.

Diagnosis is based on awareness of the complication, knowledge of the anatomy, a careful history taking and thorough physical examination followed by investigations such as MRI and electrodiagnostic studies.

Treatment is multimodal in nature with physical and pharmacological agents being used.

Preventive measures are the most important. Mention of this complication in the pre-operative discussion and consent forms will be helpful. All involved personnel must be made aware of this complication and the preventive measures to be adopted along with constant monitoring of the patient and risk factors.

\section{REFERENCES}

1. Stangl R, Altendorf-Hofmann A, von der Emde J. Brachial plexus lesions following median sternotomy in cardiac surgery. Thorac Cardiovasc Surg 1991;39:360-4. 
2. Romanowski L, Reich H, McGlynn F, Adelson MD, Taylor PJ. Brachial plexus neuropathies after advanced laparoscopic surgery. Fertil Steril 1993;60:729-32.

3. Barnett JC, Hurd WW, Rogers RM Jr, Williams NL, Shapiro SA. Laparoscopic positioning and nerve injuries. J Minim Invasive Gynecol 2007;14:664-72.

4. Gagnon J, Poulin EC. Beware of the Trendelenburg position during prolonged laparoscopic procedures. Can J Surg 1993;36:505-6.

5. Slocum HC, O'neal KC, Allen CR. Neurovascular complications from malposition on the operating table. Surg Gynecol Obstet 1948;86:729-34.

6. Brunette KE, Hutchinson DO, Ismail H. Bilateral brachial plexopathy following laparoscopic bariatric surgery. Anaesth Intensive Care 2005;33:812-5.

7. Chin P, Poole G. Bilateral brachial plexus injury during laparoscopic sigmoid colectomy. ANZ J Surg 2003;73:86-8.

8. Jackson L, Keats AS. Mechanism of brachial plexus palsy following anesthesia. Anesthesiology 1965;26:190-4.

9. Schwartz DM, Sestokas AK, Hilibrand AS, Vaccaro AR, Bose B, $\mathrm{Li} \mathrm{M}$, et al. Neurophysiological identification of position-induced neurologic injury during anterior cervical spine surgery. J Clin Monit Comput 2006;20:437-44.

10. Lachman E, Rosenberg P, Gino G, Levine S, Goldberg S, Borstein M. Axonal damage to the left musculocutaneous nerve of the left biceps muscle during laparoscopic surgery. J Am Assoc Gynecol Laparosc 2001;8:453-5.

11. Rose MA, Kam PC. Gabapentin: Pharmacology and its use in pain management. Anaesthesia 2002;57:451-62.

12. World Health Organisation. (website) . WHOs pain ladder. Geneva, Switz: World Health Organization; 2010. Available from: www.who.int/cancer/palliative/painladder/en/. Accessed 2014 August 11.

13. Watanabe S, Bruera E. Corticosteroids as adjuvant analgesics. J Pain Symptom Manage 1994;9:442-5.

14. Ben-David B, Stahl S. Prognosis of intraoperative brachial plexus injury: A review of 22 cases. Br J Anaesth 1997;79: 440-5.

15. Shveiky D, Aseff JN, Iglesia CB. Brachial plexus injury after laparoscopic and robotic surgery. J Minim Invasive Gynecol 2010;17:414-20.

16. Klauschie J, Wechter ME, Jacob K, Zanagnolo V, Montero R, Magrina $\mathrm{J}$, et al. Use of anti-skid material and patientpositioning to prevent patient shifting during robotic-assisted gynecologic procedures. J Minim Invasive Gynecol 2010;17: 504-7.

17. Luginbuhl A, Schwartz DM, Sestokas AK, Cognetti D, Pribitkin E. Detection of evolving injury to the brachial plexus during transaxillary robotic thyroidectomy. Laryngoscope 2012;122:1 10-5.

18. Karna ST, Pandey CK, Pandey VK, Singh A. Brachial plexus injury in live related donor hepatectomy: A chart review. J Postgrad Med 2014;60:287-9.

19. Thatte MR. Monitoring to prevent brachial plexus injury. J Postgrad Med 2014;60:241-2.

How to cite this article: Thomas J. Post-operative brachial plexus neuropraxia: A less recognised complication of combined plastic and laparoscopic surgeries. Indian J Plast Surg 2014;47:460-4.

Source of Support: Nil, Conflict of Interest: None declared. 